\title{
The boundary degeneracy of a singular diffusion equation
}

\author{
Huashui Zhan ${ }^{1 *}$ and Qingmei Xie ${ }^{2}$
}

${ }^{*}$ Correspondence:

huashuizhan@163.com;

2012111007@xmut.edu.cn

'School of Applied Mathematics,

Xiamen University of Technology,

Xiamen, 361024, P.R. China

Full list of author information is

available at the end of the article

\begin{abstract}
We consider the following singular diffusion equation with boundary degeneracy: $\frac{\partial u}{\partial t}=\operatorname{div}\left(d^{\alpha} \cdot|\nabla u|^{p-2} \nabla u\right),(x, t) \in Q_{T}=\Omega \times(0, T)$, where $\Omega \subset R^{N}$ is a bounded domain with appropriately smooth boundary, $p>1, \alpha>0$, and $d=d(x)=\operatorname{dist}(x, \partial \Omega)$. Though its diffusion coefficient vanishes on the boundary, it is still possible that the heat flux transfers across the boundary (Yin and Wang in Chin. Ann. Math., Ser. B 25:175-182, 2004), and it is not possible to define the homogeneous boundary value condition as usual. In the paper, under the assumption on the uniqueness of the weak solution, if the point $x$ lies in the interior of the domain $\Omega$, the paper obtains the result that the weak solution of the quoted equation has the same regular properties as those of the weak solution to the usual evolutionary $p$-Laplacian equation. However, if the point $x$ lies on the boundary $\partial \Omega$, the situation may be different. The most significant feature of the paper is that the definition of the homogeneous boundary value condition of the above equation is given. Then, if $\alpha \geq 1$, the bounded estimates of the weak solution are got by constructing the special barrier functions, and at last, how the diffusion coefficient $d^{\alpha}$ affects the gradient of the solution near the boundary is discussed.
\end{abstract}

MSC: $35 \mathrm{~K} 55 ; 35 \mathrm{~K} 65 ; 35 \mathrm{~B} 40$

Keywords: boundary degeneracy; diffusion equation; regular property; barrier function; gradient

\section{Introduction}

Consider the following singular diffusion equation with boundary degeneracy:

$$
\frac{\partial u}{\partial t}=\operatorname{div}\left(d^{\alpha} \cdot|\nabla u|^{p-2} \nabla u\right), \quad(x, t) \in Q_{T}=\Omega \times(0, T),
$$

where $\Omega \subset R^{N}$ is a bounded domain with appropriately smooth boundary, $p>1, \alpha>0$, and $d=d(x)=\operatorname{dist}(x, \partial \Omega)$ is the distance function from the boundary. If $\alpha=0$, then (1.1) becomes the following evolutionary $p$-Laplacian equation:

$$
\frac{\partial u}{\partial t}=\operatorname{div}\left(|\nabla u|^{p-2} \nabla u\right), \quad(x, t) \in Q_{T}=\Omega \times(0, T) .
$$

Equation (1.2) reflects the more practical process of heat conduction than the classical heat conduction equation $u_{t}=\Delta u$ does. For example, when $p>2$, the solution of the equation may possess the property of propagation of finite speed, while $u_{t}=\Delta u$ always has the

\section{Springer}

(02014 Zhan and Xie; licensee Springer. This is an Open Access article distributed under the terms of the Creative Commons Attribution License (http://creativecommons.org/licenses/by/2.0), which permits unrestricted use, distribution, and reproduction in any medium, provided the original work is properly cited. 
property of propagation of infinite speed which seems clearly contrary to the practice. There is a tremendous amount of related work for (1.2), one is referred to the books [1-3] etc. and the references therein.

For (1.1), the diffusion coefficient depends on the distance to the boundary. Since the diffusion coefficient vanishes on the boundary, it seems that there is no heat flux across the boundary. However, [4] has shown that the fact might not coincide with what we imagine to be the case. In fact, the exponent $\alpha$, which characterizes the vanishing ratio of the diffusion coefficient near the boundary, does determine the behavior of the heat transfer near the boundary. Let us give the definition of weak solution for (1.1) as follows.

Definition 1.1 If the function $u(x, t)$ satisfies $u \in C\left(0, T ; L^{2}(\Omega)\right) \cap L^{\infty}\left(Q_{T}\right)$, $\frac{\partial u}{\partial t} \in L^{2}\left(Q_{T}\right)$, $d^{\alpha}|\nabla u|^{p} \in L^{1}\left(Q_{T}\right)$, and for any test function $\varphi \in C_{0}^{\infty}\left(Q_{T}\right)$, the following integral equality holds:

$$
\iint_{Q_{T}}\left(\frac{\partial u}{\partial t} \varphi+d^{\alpha} \cdot|\nabla u|^{p-2} \nabla u \cdot \nabla \varphi\right) d x d t=0
$$

then the function $u$ is said to be a weak solution of (1.1).

According to [4], if $0<\alpha<p-1$, we can impose the Dirichlet boundary value condition as usual

$$
u(x, t)=g(x, t), \quad(x, t) \in \partial \Omega \times(0, T) .
$$

Otherwise, if $\alpha \geq p-1$, then the heat conduction of (1.1) is entirely free from the limitations of the boundary condition. In other words, the problem of heat conduction is entirely controlled by the initial value condition

$$
u(x, 0)=u_{0}(x), \quad x \in \Omega .
$$

This fact makes us consider whether the properties of the solutions, such as the regularity, the large time behavior etc. of (1.1) are the same as the corresponding properties of the solutions of (1.2) or not.

In this paper, under the assumption of the uniqueness of the solution to the singular diffusion (1.1), Section 2 discusses the regular properties of the solution of (1.1) in the interior points of $Q_{T}$ by using the method as the Chapter 2 of [1]. Section 3 first gives the definition of the homogeneous boundary value condition of (1.1), then by using some ideas of [5], if $1 \leq \alpha$, the boundedness estimates of the weak solution of (1.1) on the boundary are obtained. In the last section of the paper, we emphasize the analysis of how the diffusion coefficient $d^{\alpha}$ affects the gradient of the solution near the boundary in some cases.

Remark 1.2 If the initial value $u_{0}(x)$ satisfies

$$
u_{0}(x) \in L^{\infty}(\Omega), \quad d^{\alpha}\left|\nabla u_{0}\right|^{p} \in L^{1}(\Omega),
$$

then the results of [4] have shown that the uniqueness of the solution to (1.1) is true, only if $\alpha>0$. The existence and the uniqueness of the solution to a more general equation than (1.1) had been studied in [6]. 


\section{Estimate in the interior of the domain}

We first introduce the following lemmas from [1].

Lemma 2.1 There exists a constant $\gamma$ only depending on $p, q, N$ such that for any $v \in$ $V_{0}^{q, p}\left(\Omega_{T}\right)$ and $h=\frac{p(q+N)}{N}$,

$$
\iint_{\Omega_{T}}|v(x, t)|^{h} d x d t \leq \gamma\left(\iint_{\Omega_{T}}|\nabla v(x, t)|^{p} d x d t\right) \cdot\left(\operatorname{ess} \sup \int_{\Omega}|v(x, t)|^{q} d x\right)^{\frac{p}{N}},
$$

where $V_{0}^{q, p}\left(\Omega_{T}\right)$ is the closure of $C_{0}^{\infty}\left(\Omega_{T}\right)$ in space of $V^{q, p}\left(\Omega_{T}\right)$.

Lemma 2.2 Let $Q_{n}(n=1,2, \ldots)$ be a sequence of bounded open sets in $Q_{T}, Q_{n+1} \subset Q_{n}$. Assume for any $q \geq 1, v \in L^{q}\left(Q_{T}\right)$ and that there exist some constants $\alpha_{0} \geq 0, \lambda, C_{0}, C_{1}>0$, $K>1$, such that the following inequality holds:

$$
\iint_{Q_{n+1}}|v|^{\alpha_{0}+\lambda K^{n+1}} d x d t \leq\left(C_{0} C_{1}^{n} \iint_{Q_{n}}|v|^{\alpha_{0}+\lambda K^{n}} d x d t\right)^{K}
$$

Then

$$
\operatorname{ess} \sup _{Q_{n}}|v| \leq\left(C_{0}^{\frac{K}{K-1}} \bar{C}_{1} \iint_{Q_{n_{0}}}|v|^{\alpha_{0}+\lambda K^{n_{0}}} d x d t\right)^{\frac{1}{\lambda K^{n_{0}}}}
$$

holds, where $\bar{C}_{1}=C_{1}^{K_{1}}, K_{1}=\sum_{n=n_{0}}^{\infty} n K^{-\left(n-n_{0}\right)}$ and $n_{0} \in N^{+}$.

By the above two lemmas, we are able to get the following theorem.

Theorem 2.3 Let $\Omega$ be a uniformly $C^{1}$ domain. If $u$ is the unique solution of (1.1) in $Q_{T}$, and $p>\max \left\{1, \frac{2 N}{N+1}\right\}$, then

$$
\frac{\partial u}{\partial x_{i}} \in L_{\mathrm{loc}}^{\infty}\left(Q_{T}\right), \quad i=1,2, \ldots, N
$$

The proof of the theorem is just similar to that Proposition 4.1 in Chapter 2 of [1], in which the same conclusion on (1.2) is obtained.

Proof Since $u$ is the unique solution of (1.1) in $Q_{T}$, we can assume that $u$ is the limit of $u_{n}$, which is the classical solution of the following regularized problem:

$$
\begin{aligned}
& \frac{\partial u}{\partial t}=\operatorname{div}\left[\left(d^{\alpha}+\frac{1}{n}\right)\left(|\nabla u|^{2}+\frac{1}{n}\right)^{\frac{p-2}{2}} \nabla u\right], \quad(x, t) \in Q_{T}=\Omega \times(0, T), \\
& u(x, 0)=u_{0, n}(x), \quad x \in \Omega, \\
& u(x, t)=g(x, t), \quad(x, t) \in \partial \Omega \times(0, T),
\end{aligned}
$$

where $u_{0, n}(x)$ is the smoothly mollified functions of $u_{0}(x)$. Let $K \subset Q_{T}$ be an any compact set. Similar to the proof of Lemma 2.3 in the Chapter 2 of [1], which discusses (1.2), we are 
able to get

$$
\iint_{K}\left|\nabla u_{n}\right|^{q} d x d t \leq C(q, K, p, N)
$$

Denote $B_{\rho}\left(x_{0}\right)=\left\{x:\left|x-x_{0}\right|<\rho\right\}$, and for simplicity, denote $u_{n}$ as $u$.

Now, we differentiate (2.2) with respect to $x_{j}$, and we obtain

$$
\frac{\partial u_{x_{j}}}{\partial t}=\left[\left(d+\frac{1}{n}\right)^{\alpha}\left(|\nabla u|^{2}+\frac{1}{n}\right)^{\frac{p-2}{2}} u_{x_{i}}\right]_{x_{i} x_{j}} .
$$

Let

$$
\begin{aligned}
& T_{n}=\frac{t_{0}}{2}-\frac{t_{0}}{2^{n+2}}, \quad \rho_{n}=\rho+\frac{\rho}{2^{n}}, \\
& \bar{\rho}_{n}=\frac{1}{2}\left(\rho_{n}+\rho_{n+1}\right)=\rho+\frac{3 \rho}{2^{n+2}}, \\
& B_{n}=B_{\rho_{n}}\left(x_{0}\right), \quad B_{n}^{\prime}=B_{\overline{\rho_{n}}}\left(x_{0}\right), \\
& Q_{n}=B_{n} \times\left(T_{n}, T\right), \quad Q_{n}^{\prime}=B_{n}^{\prime} \times\left(T_{n+1}, T\right) .
\end{aligned}
$$

Assume that $\xi_{n}$ is the cut off of functions smoothly in $Q_{n}, \xi_{n}(\cdot, t) \in C_{0}^{1}\left(B_{n}\right), \xi_{n}=0, \forall t \leq T_{n}$, $\xi_{n}=1, \forall(x, t) \in Q_{n}^{\prime},\left|\nabla \xi_{n}\right| \leq \frac{2^{n+2}}{\rho}, 0 \leq \xi_{n t} \leq \frac{2^{n+3}}{t_{0}}$.

Let $x_{0} \in \Omega, B_{4 \rho}\left(x_{0}\right)=B_{4 \rho} \subset \Omega$. We choose $v=|\nabla u|^{2}+\frac{1}{n}$, multiply the two sides of (2.6) with $\xi_{n}^{2} v^{\beta} u_{x_{j}}$, and integrate on $B_{2 \rho} \times\left(\frac{t_{0}}{4}, t\right)$. Then we can obtain the following equality:

$$
\begin{aligned}
& \frac{1}{2(\beta+1)} \int_{\frac{t_{0}}{4}}^{t} \int_{B_{2 \rho}} \xi_{n}^{2} \nu^{\beta+1}(x, t) d x d t+\int_{\frac{t_{0}}{4}}^{t} \int_{B_{2 \rho}} \xi_{n}^{2}\left(d+\frac{1}{n}\right)^{\alpha}\left(v^{\frac{p-2}{2}} u_{x_{i}}\right)_{x_{j}}\left(v^{\beta} u_{x_{j}}\right)_{x_{i}} d x d t \\
& =-\alpha \int_{\frac{t_{0}}{4}}^{t} \int_{B_{2 \rho}} \xi_{n}^{2} d_{x_{j}}\left(d+\frac{1}{n}\right)^{\alpha-1} v^{\frac{p-2}{2}} u_{x_{i}}\left(v^{\beta} u_{x_{j}}\right)_{x_{i}} d x d t \\
& \quad-2 \int_{\frac{t_{0}}{4}}^{t} \int_{B_{2 \rho}} \xi_{n} \xi_{n x_{i}}\left(d+\frac{1}{n}\right)^{\alpha}\left(v^{\frac{p-2}{2}} u_{x_{i}}\right)_{x_{j}} v^{\beta} u_{x_{j}} d x d t \\
& \quad-2 \alpha \int_{\frac{t_{0}}{4}}^{t} \int_{B_{2 \rho}} \xi_{n} \xi_{n x_{i}} d_{x_{j}}\left(d+\frac{1}{n}\right)^{\alpha-1} v^{\frac{p-2}{2}} u_{x_{i}} v^{\beta} u_{x_{j}} d x d t \\
& +\alpha \int_{\frac{t_{0}}{4}}^{t} \int_{B_{2 \rho}} \xi_{n}^{2} \nu^{\beta} u_{x_{j}}\left[\left(d+\frac{1}{n}\right)^{\alpha-1} d_{x_{j}} v^{\frac{p-2}{2}} u_{x_{i}}\right] d x d t \\
& +\frac{1}{\beta+1} \int_{\frac{t_{0}}{4}}^{t} \int_{B_{2 \rho}} \xi_{n} \xi_{n t} v^{\beta+1} d x d t
\end{aligned}
$$

Using the fact of that, according to [7], the distance function always satisfies $|\nabla d|=1$ in the sense of distribution, and clearly

$$
\left|d_{x_{j}}\right| \leq|\nabla d|=1, \quad \forall x \in \Omega
$$

By the Young inequality,

$$
v^{\frac{p-2}{2}} u_{x_{i}} \nu^{\beta-1} v_{x_{i}} u_{x_{j}} \leq \varepsilon v^{\frac{p+2 \beta-4}{2}}|\nabla v|^{2}+c(\varepsilon) v^{\frac{p+2 \beta}{2}},
$$


Zhan and Xis Journal of Inequalities and Applications 2014, 2014:284

Page 5 of 20

http://www.journalofinequalitiesandapplications.com/content/2014/1/284

$$
\begin{aligned}
& v^{\frac{p-2}{2}} u_{x_{i}} v^{\beta} u_{x_{i} x_{j}} \leq \varepsilon v^{\frac{p+2 \beta-2}{2}} u_{x_{i} x_{j}} u_{x_{i} x_{j}}+c(\varepsilon) v^{\frac{p+2 \beta}{2}} \\
& \xi_{n x_{i}} v^{\beta} u_{x_{j}}\left(v^{\frac{p-2}{2}} u_{x_{i}}\right)_{x_{j}} \leq \varepsilon\left|\nabla \xi_{n}\right|^{2} v^{\frac{p+2 \beta}{2}}+\varepsilon v^{\frac{p+2 \beta-4}{2}}|\nabla v|^{2}+\varepsilon v^{\frac{p+2 \beta-2}{2}} u_{x_{i} x_{j}} u_{x_{i} x_{j}},
\end{aligned}
$$

and

$$
\begin{aligned}
\left(v^{\frac{p-2}{2}} u_{x_{i}}\right)_{x_{j}}\left(v^{\beta} u_{x_{j}}\right)_{x_{i}}= & v^{\frac{p+2 \beta-2}{2}} u_{x_{i} x_{j}} u_{x_{i} x_{j}}+\frac{p+2 \beta-2}{4} v^{\frac{p+2 \beta-4}{2}}|\nabla v|^{2} \\
& +\frac{\beta(p-2)}{2} v^{\frac{p+2 \beta-6}{2}}(\nabla u \cdot \nabla v)^{2} .
\end{aligned}
$$

We obtain

$$
\begin{aligned}
& \frac{1}{2(\beta+1)} \int_{\frac{t_{0}}{4}}^{t} \int_{B_{2 \rho}} \xi_{n}^{2} v^{\beta+1}(x, t) d x+(1-c \varepsilon) \int_{\frac{t_{0}}{4}}^{t} \int_{B_{2 \rho}} \xi_{n}^{2} v^{\frac{p+2 \beta-2}{2}} u_{x_{i} x_{j}} u_{x_{i} x_{j}} d x d t \\
& \quad+\left(\frac{p+2 \beta-2}{4}-c \varepsilon\right) \int_{\frac{t_{0}}{4}}^{t} \int_{B_{2 \rho}} v^{\frac{p+2 \beta-4}{2}}|\nabla v|^{2} d x d t \\
& \quad+\frac{\beta(p-2)}{2} \int_{\frac{t_{0}}{4}}^{t} \int_{B_{2 \rho}} v^{\frac{p+2 \beta-6}{2}}(\nabla u \cdot \nabla v)^{2} d x d t \\
& \leq c(\varepsilon) \int_{\frac{t_{0}}{4}}^{t} \int_{B_{2 \rho}} v^{\frac{p+2 \beta}{2}}\left(1+\left|\nabla \xi_{n}\right|^{2}\right) d x d t+\frac{c}{\beta+1} \int_{\frac{t_{0}}{4}}^{t} \int_{B_{2 \rho}} \xi_{n} v^{\beta+1} d x d t,
\end{aligned}
$$

where $\varepsilon$ is an appropriately small positive constant.

(1) When $p \geq 2$, denote

$$
w=v^{\frac{p+2 \beta}{4}}, \quad \lambda=\frac{4(\beta+1)}{p+2 \beta} .
$$

From (2.9), we can obtain

$$
\begin{aligned}
& \sup _{T_{n}<t<T} \int_{B_{n}}\left(\xi_{n}^{\frac{2}{\lambda}} w\right)^{\lambda}(x, t) d x+\iint_{Q_{n}}\left(\xi_{n}^{\frac{2}{\lambda}}|\nabla w|\right)^{2} d x d t \\
& \quad \leq C\left[\left(1+\frac{2^{2 n}}{\rho^{2}}\right) \iint_{Q_{n}} w^{2} d x d t+\frac{2^{n}}{t_{0}} \iint_{Q_{n}} w^{\lambda} d x d t\right],
\end{aligned}
$$

by Lemma 2.1 and (2.10),

$$
\iint_{Q_{n+1}} w^{2+\frac{2 \lambda}{N}} d x d \tau \leq C\left[\left(1+\frac{2^{2 n}}{\rho^{2}}\right) \iint_{Q_{n}} w^{2} d x d t+\frac{2^{n}}{t_{0}} \iint_{Q_{n}} w^{\lambda} d x d t\right]
$$

which implies that

$$
\iint_{Q_{n+1}} v^{\frac{p+2 \beta}{2}+\frac{2 \beta+2}{N}} d x d \tau \leq C\left[\left(1+\frac{2^{2 n}}{\rho^{2}}\right) \iint_{Q_{n}} v^{\frac{p+2 \beta}{2}} d x d \tau+\frac{2^{n}}{t_{0}} \iint_{Q_{n}} v^{\beta+1}\right]^{1+\frac{2}{N}},
$$


where $k=1+\frac{2}{N}$. By choosing $2 \beta=k^{n}-2$, the above formula can be changed into

$$
\begin{aligned}
& \iint_{Q_{n+1}} v^{\frac{p-2}{2}+\frac{k^{n+1}}{2}} d x d \tau \\
& \quad \leq C\left[\left(1+\frac{2^{2 n}}{\rho^{2}}\right) \iint_{Q_{n}} v^{\frac{p-2}{2}+\frac{k^{n}}{2}} d x d \tau+\frac{2^{n}}{t_{0}} \iint_{Q_{n}} v^{\frac{k^{n}}{2}} d x d \tau\right]^{k} .
\end{aligned}
$$

If

$$
\frac{2^{n}}{t_{0}} \iint_{Q_{n}} v^{\frac{k^{n}}{2}} d x d \tau \geq\left(1+\frac{2^{2 n}}{\rho^{2}}\right) \iint_{Q_{n}} v^{\frac{p-2}{2}+\frac{k^{n}}{2}} d x d \tau
$$

then, by the Hölder inequality, we see that

$$
\iint_{Q_{n}} v^{\frac{p-2}{2}+\frac{k^{n}}{2}} d x d \tau \leq\left(\frac{\rho^{2}}{t_{0}}\right)^{\frac{\left(p-2+k^{n}\right)}{(p-2)}} \operatorname{mes} Q_{n},
$$

which implies that

$$
\sup _{B_{\rho} \times\left(\frac{t_{0}}{2}, T\right)} v \leq\left(\frac{\rho^{2}}{t_{0}}\right)^{\frac{2}{p-2}}
$$

Then we obtain the theorem.

If (2.12) is not true, we have

$$
\iint_{Q_{n+1}} v^{\frac{p-2}{2}+\frac{k^{n+1}}{2}} d x d \tau \leq C\left[\left(1+\frac{2^{2 n}}{\rho^{2}}\right) \iint_{Q_{n}} v^{\frac{p-2}{2}+\frac{k^{n}}{2}} d x d \tau\right]^{k}
$$

By Lemma 2.2,

$$
\sup _{Q_{n}} v \leq C \rho^{-(N+2)} \iint_{Q_{n_{0}}} v^{\frac{p-2}{2}+\frac{k^{n} 0}{2}} d x d \tau
$$

where $n_{0}$ is a positive integer which makes $k^{n_{0}}>2$ hold. Then we can obtain Theorem 2.3 according to (2.5). Therefore, when $p>2$,

$$
\frac{\partial u}{\partial u_{i}} \in L_{\mathrm{loc}}^{\infty}\left(Q_{T}\right)
$$

(2) When $p<2$, we can get

$$
v^{\frac{p+2 \beta-2}{2}} \sum_{j=1}^{N}\left|\nabla u_{x_{j}}\right|^{2} \geq \frac{1}{4} v^{\frac{p+2 \beta-4}{2}}|\nabla v|^{2}
$$

and

$$
\begin{aligned}
& \frac{\beta(p-2)}{2} \int_{0}^{t_{0}} \int_{B_{2 \rho}} \xi_{n}^{2} \nu^{\frac{p+2 \beta-6}{2}}(\nabla u \cdot \nabla v)^{2} d x d \tau \\
& \geq \frac{\beta(p-2)}{2} \int_{0}^{t_{0}} \int_{B_{2 \rho}} \xi_{n}^{2} \nu^{\frac{p+2 \beta-4}{2}}|\nabla u|^{2} d x d \tau .
\end{aligned}
$$


Then according to (2.9),

$$
\begin{aligned}
& \sup _{\frac{t_{0}}{4}<t<T} \int_{B_{2 \rho}} \xi_{n}^{2} \nu^{\beta+1}(x, \tau) d x+\iint_{Q\left(2 \rho, \frac{t_{0}}{4}\right)} \xi_{n}^{2} \nu^{\alpha_{p}}|\nabla u|^{2} d x d \tau \\
& \quad \leq C \iint_{Q\left(2 \rho, \frac{t_{0}}{4}\right)} \nu^{\alpha_{p}+2}\left|\nabla \xi_{n}\right|^{2} d x d \tau+C \iint_{Q\left(2 \rho, \frac{t_{0}}{4}\right)} \xi_{n} \xi_{n t} \nu^{\beta+1} d x d \tau,
\end{aligned}
$$

where $\alpha_{p}=\frac{p+2 \alpha-4}{2}$. Then using Lemma 2.1, similar to the discussion of the case (1), we can obtain

$$
\begin{aligned}
& {\left[\iint_{Q_{n+1}} v^{\frac{N(2-p)}{4}+\frac{k^{n+1}}{2}} d x d \tau\right]^{\frac{1}{k}}} \\
& \quad \leq C\left[\frac{2^{2 n}}{t_{0}} \iint_{Q_{n}} v^{\frac{N(2-p)}{4}+\frac{k^{n}}{2}} d x d \tau+\left(1+\frac{2^{2 n}}{\rho^{2}}\right) \iint_{Q_{n}} v^{\frac{(N-2)(2-p)}{4}+\frac{k^{n}}{2}} d x d \tau\right] .
\end{aligned}
$$

If

$$
\left(1+\frac{2^{2 n}}{\rho^{2}}\right) \iint_{Q_{n}} v^{\frac{(N-2)(2-p)}{4}+\frac{k^{n}}{2}} d x d \tau \geq \frac{2^{2 n}}{t_{0}} \iint_{Q_{n}} v^{\frac{N(2-p)}{4}+\frac{k^{n}}{2}} d x d \tau
$$

then by the Hölder inequality, we have

$$
\iint_{Q_{n}} v^{\frac{N(2-p)}{4}+\frac{k^{n}}{2}} d x d \tau \leq\left(\frac{t_{0}+\rho^{2}}{\rho^{2}}\right)^{\frac{\left[N(2-p)+k^{n}\right]}{(2-p)}} \operatorname{mes} Q_{n},
$$

which implies that

$$
\sup _{B_{\rho} \times\left(\frac{t_{0}}{2}, T\right)} v \leq\left(\frac{t_{0}}{\rho^{2}}\right)^{\frac{2}{2-p}} .
$$

If (2.16) is not true, then from (2.15), we have

$$
\left[\iint_{Q_{n+1}} v^{\frac{N(2-p)}{4}+\frac{k^{n+1}}{2}} d x d \tau\right]^{\frac{1}{k}} \leq \gamma\left(1+\frac{2^{2 n}}{\rho^{2}}\right) \iint_{Q_{n}} v^{N(2-p)+\frac{k^{n}}{2}} d x d \tau .
$$

Then using Lemma 2.2, we obtain

$$
\sup _{B_{\rho} \times\left(\frac{t_{0}}{2}, T\right)} v \leq C\left[\rho^{-(N+2)} \iint_{Q_{n_{0}}} v^{\frac{N(2-p)}{4}+\frac{k^{n_{0}}}{2}} d x d \tau\right]^{\frac{2}{k^{n_{0}}}} .
$$

Also we can obtain Theorem 2.3 according to (2.5).

Therefore when $p<2$, also

$$
\frac{\partial u}{\partial u_{i}} \in L_{\mathrm{loc}}^{\infty}\left(Q_{T}\right)
$$

The proof of Theorem 2.3 is complete. 
Theorem 2.4 Supposed that $u$ is a weak solution of (1.1) in $Q_{T}$, then for any compact set $K \subset Q_{T},\left(x_{1}, t_{1}\right),\left(x_{2}, t_{2}\right) \in K$,

$$
\left|u\left(x_{1}, t_{1}\right)-u\left(x_{2}, t_{2}\right)\right| \leq c\left(\left|x_{1}-x_{2}\right|+\left|t_{1}-t_{2}\right|^{\frac{1}{2}}\right),
$$

where $c$ is a constant only dependent on $N, p, d(K, \partial \Omega),\|u\|_{L^{\infty}(K)}$.

The proof of the theorem is just similar to that of Theorem 4.3 in Chapter 2 of [1], in which the same conclusion on (1.2) is obtained.

Proof We only need to prove that $u$ satisfies (2.17) in $B_{R} \times\left(t_{0}, T\right)$, for any $R>0$ such that $B_{R} \subset \Omega, t_{0} \in(0, T)$. Let $u_{\varepsilon}$ be the usual mollified function of $u$,

$$
u_{\varepsilon}(x, t)=J_{\varepsilon} * u(x, t)=\int_{0}^{T} \int_{R^{N}} j_{\varepsilon}(x-y, t-\tau) u(y, \tau) d y d \tau,
$$

where $0<\varepsilon<t_{0}<t<T-\varepsilon$. Then for any $x_{1}, x_{2} \in B_{R}$, according to the definition of $u_{\varepsilon}(x, t)$, we easily get

$$
\begin{aligned}
& u_{\varepsilon}\left(x_{1}, t\right)-u_{\varepsilon}\left(x_{2}, t\right) \\
& \quad=\int_{0}^{T} \int_{R^{N}} \int_{0}^{1} j_{\varepsilon}\left(s x_{1}+(1-s) x_{2}-y, t-\tau\right) \nabla_{y} u(y, \tau) d s d y d \tau\left(x_{1}-x_{2}\right) .
\end{aligned}
$$

According to Theorem 2.3, we have

$$
\begin{aligned}
& \left|u_{\varepsilon}\left(x_{1}, t\right)-u_{\varepsilon}\left(x_{2}, t\right)\right| \\
& \quad \leq \int_{0}^{T} \int_{R^{N}} \int_{0}^{1}\left|j_{\varepsilon}\left(s x_{1}+(1-s) x_{2}-y, t-\tau\right)\right|\left|\nabla_{y} u(y, \tau)\right| d s d y d \tau\left|x_{1}-x_{2}\right| \\
& \quad \leq c\left|x_{1}-x_{2}\right|
\end{aligned}
$$

here and in what follows $c$ is a constant independent of $\varepsilon$.

Now, let $0<\varepsilon<t_{0}<t_{1}<t_{2}<T, B(\Delta t)=B_{(\Delta t)^{\frac{1}{2}}}\left(x_{0}\right), \varphi \in C_{0}^{1}(B(\Delta t)), x_{0} \in B_{R}, \Delta t=t_{2}-t_{1}$. Then, similar to Chapter 2 of [1], we have

$$
\begin{aligned}
& \int_{B(\Delta t)} \varphi(x)\left[u_{\varepsilon}\left(x, t_{2}\right)-u_{\varepsilon}\left(x, t_{1}\right)\right] d x \\
& \quad=-\Delta t \int_{B(\Delta t)} \varphi(x) \int_{0}^{1} \int_{0}^{T} \int_{R^{N}} j_{\varepsilon \tau}\left(x-y, s t_{2}+(1-s) t_{1}-\tau\right) u(y, \tau) d y d \tau d s d x .
\end{aligned}
$$

For fixed $(x, t) \in Q_{t}, 0<\varepsilon<t_{0}<t<T-\varepsilon, J_{\varepsilon}(x-y, t-\tau) \in C_{0}^{1}\left(Q_{T}\right)$. Let us choose the test function in the definition of generalized solution (1.3) as $\varphi(x)=J_{\varepsilon}(x-y, t-\tau)$. Then we have

$$
\begin{aligned}
& \int_{0}^{T} \int_{R^{N}} j_{\varepsilon \tau}\left(x-y, s t_{2}+(1-s) t_{1}-\tau\right) u(y, \tau) d y d \tau \\
& \quad=\int_{0}^{T} \int_{R^{N}} d^{\alpha} \cdot\left|\nabla_{y} u\right|^{p-2} \nabla_{y} u \nabla_{y} j_{\varepsilon}\left(x-y, s t_{2}+(1-s) t_{1}-\tau\right) d y d \tau .
\end{aligned}
$$


By (2.19), we obtain

$$
\begin{aligned}
& \int_{B(\Delta t)} \varphi(x)\left[u_{\varepsilon}\left(x, t_{2}\right)-u_{\varepsilon}\left(x, t_{1}\right)\right] d x \\
& =-\Delta t \int_{B(\Delta t)} \varphi(x) \int_{0}^{1} \int_{0}^{T} \int_{R^{N}} d^{\alpha} \cdot\left|\nabla_{y} u\right|^{p-2} \nabla_{y} u \nabla_{y} j_{\varepsilon}(x-y, \\
& \left.s t_{2}+(1-s) t_{1}-\tau\right) d y d \tau d s d x \\
& =-\Delta t \int_{0}^{1} \int_{B(\Delta t)} \nabla_{x} \varphi J_{\varepsilon}\left(d^{\alpha} \cdot\left|\nabla_{y} u\right|^{p-2} \nabla_{y} u\right)\left(x, s t_{2}+(1-s) t_{1}\right) d x d s .
\end{aligned}
$$

Let $\delta(s) \in C_{0}^{1}(R), \delta(s) \geq 0, \int_{R} \delta(s) d s=1$, when $s \geq 1, \delta(s)=0$. For any $h>0$, we define $\delta_{h}(s)=\frac{\delta\left(\frac{s}{h}\right)}{h}$. By the approximation process, we know that (2.20) is also true for any $\varphi \in$ $W_{0}^{1,1}(B(\Delta t))$. Choosing $\varphi=\varphi_{h}(x)=\int_{-h}^{(\Delta t)^{\frac{1}{2}}-\left|x-x_{0}\right|-2 h} \delta_{h}(s) d s$ in (2.20), then

$$
\begin{aligned}
\int_{B(\Delta t)} \varphi_{h}(x)\left(u_{\varepsilon}\left(x, t_{2}\right)-u_{\varepsilon}\left(x, t_{1}\right)\right) d x \\
=-\Delta t \int_{0}^{1} \int_{B(\Delta t)} \delta_{h}\left((\Delta t)^{\frac{1}{2}}-\left|x-x_{0}\right|-2 h\right) \cdot \frac{x_{0 i}-x_{i}}{\left|x-x_{0}\right|} \\
\cdot J_{\varepsilon}\left(d^{\alpha} \cdot\left|\nabla_{y} u\right|^{p-2} \nabla_{y} u\right)\left(x, s t_{2}+(1-s) t_{1}\right) d x d s .
\end{aligned}
$$

We notice that, when $x \in B(\Delta t), \lim _{h \rightarrow 0} \varphi_{h}(x)=0$. But $\delta_{h}\left((\Delta t)^{\frac{1}{2}}-\left|x-x_{0}\right|-2 h\right)=0$ holds when $\left|x-x_{0}\right|<(\triangle t)^{\frac{1}{2}}-h$; then $\delta_{h} \leq \frac{c}{h}$ and

$$
\operatorname{mes}\left(B(\triangle t) \backslash B_{(\triangle t)^{\frac{1}{2}}-h}\left(x_{0}\right)\right) \leq \operatorname{ch}(\triangle t)^{\frac{N-1}{2}} .
$$

Therefore using Theorem 2.3 and (2.21), letting $h \rightarrow 0$, we obtain

$$
\left|\int_{B(\triangle t)}\left[u_{\varepsilon}\left(x, t_{2}\right)-u_{\varepsilon}\left(x, t_{1}\right)\right] d x\right| \leq c(\Delta t)^{\frac{N+1}{2}} \text {. }
$$

By the mean value theorem, there exists $x^{*} \in B(\triangle t)$, such that

$$
\left|u_{\varepsilon}\left(x^{*}, t_{2}\right)-u_{\varepsilon}\left(x^{*}, t_{1}\right)\right| \leq c(\Delta t)^{\frac{1}{2}}
$$

Noticing that

$$
\begin{aligned}
& \left|u_{\varepsilon}\left(x_{0}, t_{2}\right)-u_{\varepsilon}\left(x_{0}, t_{1}\right)\right| \\
& \quad \leq\left|u_{\varepsilon}\left(x_{0}, t_{2}\right)-u_{\varepsilon}\left(x^{*}, t_{2}\right)\right|+\left|u_{\varepsilon}\left(x^{*}, t_{2}\right)-u_{\varepsilon}\left(x^{*}, t_{1}\right)\right|+\left|u_{\varepsilon}\left(x^{*}, t_{1}\right)-u_{\varepsilon}\left(x_{0}, t_{1}\right)\right| \\
& \quad \leq c(\triangle t)^{\frac{1}{2}}
\end{aligned}
$$

combining this formula with (2.18), letting $\varepsilon \rightarrow 0$, we obtain the desired result.

By Theorems 2.3 and 2.4, similar to Chapter 2 of [1], it is not difficult to prove the following theorem, and we omit the details here. 
Theorem 2.5 Let $p>\max \left\{1, \frac{2 N}{N+2}\right\}$, $u$ is the generalized solution of (1.1) in $Q_{T}$, then $u_{x_{j}}$ $(j=1,2, \ldots, N)$ is locally Hölder continuous in $Q_{T}$.

\section{Estimates on the boundary}

Now, we assume that the boundary $\partial \Omega$ is of class $C^{2}$. That is, there exists a number $\rho_{0} \in(0,1)$ such that for all $x_{0} \in \partial \Omega$ the portion of $\partial \Omega$ within the ball $B_{\rho_{0}}\left(x_{0}\right)$ can be represented, in a local system of coordinates, as the graph of a $C^{2}$ function $\varphi^{\left(x_{0}\right)}$ such that $\varphi^{\left(x_{0}\right)}\left(x_{0}\right)=0$, and for $x \in B_{\rho_{0}}\left(x_{0}\right) \cap \Omega=\left\{x=\left(x_{1}, x_{2}, \ldots, x_{N-1}, x_{N}\right): x_{N}>0\right\}, x \in B_{\rho_{0}}\left(x_{0}\right) \cap$ $\partial \Omega=\left\{x=\left(x_{1}, x_{2}, \ldots, x_{N-1}, x_{N}\right): x_{N}=0\right\}$.

Let $u$ be the unique nonnegative bounded solution of (1.1) in the sense of Definition 1.1. Then $u(x, t)$ satisfies $u \in C\left(0, T ; L^{2}(\Omega)\right) \cap L^{\infty}\left(Q_{T}\right), \frac{\partial u}{\partial t} \in L^{2}\left(Q_{T}\right), d^{\alpha}|\nabla u|^{p} \in L^{1}\left(Q_{T}\right)$. From this definition, we know that one cannot define the trace of $u$ on the boundary except for $\alpha=0$.

But the results of [4] show that if $\alpha<p-1$, one can define the trace of $u$ on the boundary, and the homogeneous boundary value condition can be defined as usual. However, if $\alpha \geq$ $p-1$, then the heat conduction of (1.1) is entirely free from the limitations of the boundary condition, in other words, the problem of heat conduction is entirely controlled by the initial value condition, then in this case one cannot give the homogeneous boundary value condition as usual. Fortunately, no matter how the diffusion coefficient $\alpha$ satisfies $0<\alpha<$ $p-1$ or $\alpha \geq p-1$, the results of [4] had shown that the uniqueness of the solution is true (cf. Remark 1.2$)$ only if the initial value $u_{0}(x)$ is suitably smooth. Then we can give the following definition.

Definition 3.1 If $u_{0}(x)$ is suitably smooth, $u$ is the limit of the solutions $\left\{u_{n}\right\}$ of the following problem:

$$
\begin{aligned}
& \frac{\partial u}{\partial t}=\operatorname{div}\left[\left(d^{\alpha}+\frac{1}{n}\right)\left(|\nabla u|^{2}+\frac{1}{n}\right)^{\frac{p-2}{2}} \nabla u\right], \quad(x, t) \in Q_{T}=\Omega \times(0, T), \\
& u(x, 0)=u_{0, n}(x), \quad x \in \Omega, \\
& u(x, t)=0, \quad(x, t) \in \partial \Omega \times(0, T),
\end{aligned}
$$

where $u_{0, n}(x)$ is the smoothly mollified functions of $u_{0}(x)$. Then we say $u$ is the solution of (1.1) with the homogeneous boundary value condition.

We will get Estimates above.

Theorem 3.2 Let $u$ be the unique nonnegative bounded solution of (1.1) with the homogeneous boundary value condition in the sense of Definition 3.1. Supposed that $1 \leq \alpha$, for $s \in(0, T)$,

$$
u(x, t) \leq M, \quad(x, t) \in \Omega \times(s, T)
$$

then

$$
u(x, t) \leq k C d(x, \partial \Omega), \quad(x, t) \in \Omega \times(s, T)
$$


where the constant $C$ depending upon $M, N, p, s$, and the constant $k$ is a constant independent of $s, M$.

The key idea of the proof is to work with cylinders whose dimensions are suitably rescaled to reflect the degeneracy exhibited by the equation. The main idea is to construct a supersolution of the equation.

Proof Fix $\left(x_{0}, t_{0}\right) \in \partial \Omega \times(s, T)$. We may assume that $\left(x_{0}, t_{0}\right) \equiv(0,0)$ and in the vicinity of $(0,0)$, after flattening of $\partial \Omega$ near $x_{0}$, without loss of generality, let us assume that $\partial \Omega$ coincides with the portion of hyperplane $\left\{x_{N}=0\right\}$, the inclusion $\Omega \cap\left\{|x|<\rho_{0}\right\} \subset\left\{x_{N}>0\right\}$. Let $y=(0, \ldots, 0,-1)$, and the set

$$
\aleph_{k}=\left\{(x, t): x_{N}>0,1<|x-y|<1+\frac{1}{k},-s_{n} \leq t \leq 0\right\} .
$$

We assume $k$ is so large that $\aleph_{k} \subset B_{\rho_{0}}^{+} \times(s, 0]$. Consider the following problem:

$$
\begin{aligned}
& \frac{\partial v}{\partial t}=\operatorname{div}\left[\left(d^{\alpha}+\frac{1}{n}\right)\left(|\nabla v|^{2}+\frac{1}{n}\right)^{\frac{p-2}{2}} \nabla v\right], \quad(x, t) \in \aleph_{k} . \\
& v\left(x,-s_{n}\right)=u_{n}\left(x,-s_{n}\right), \quad x \in B_{k}^{+}, \\
& v(x, t)=u_{n}(x, t)-\frac{1}{n}, \quad(x, t) \in \partial B_{k}^{+} \times\left[-s_{n}, 0\right],
\end{aligned}
$$

where $u_{n}$ is the solution of the problem (3.1)-(3.3), $0<s_{n}<s<T, s_{n} n$ is small enough, and $B_{k}^{+}=\left\{x: x_{N}>0,1<|x-y|<1=\frac{1}{k}\right\} \cap B_{\rho_{0}}^{+}$. By the comparison theorem [8, p.119], we have

$$
v \leq u_{n}
$$

Let us construct a barrier for $u$ in $\aleph_{k}$. Consider the function

$$
\eta_{k}(x, t)=e^{-k(|x-y|-1)} e^{t}
$$

Our barrier is given by

$$
\Psi_{k}=C M\left(1-\eta_{k}(x, t)\right)+\gamma t, \quad(x, t) \in \aleph_{k},
$$

where the constants $\gamma, C$ are to be chosen later so large that $v \leq \Psi_{k}$ on the parabolic boundary of $\aleph_{k}$. This holds true on the portion of such a boundary lying on the hyperplane $x_{N}=0$,

$$
\Psi_{k}=C M\left(1-\eta_{k}(x, t)\right)+\gamma t \geq C M\left(1-e^{-k(|x-y|-1)}\right)-\gamma s_{n} \geq-\frac{1}{n}
$$

provided that $\gamma \leq \frac{1}{n s_{n}}$. On the portion $\left\{|x-y|=1+\frac{1}{k}\right\} \cap\left\{x_{N} \geq 0\right\}$, we have

$$
\Psi_{k} \geq C M\left(1-e^{-1+t}\right)-\gamma s_{n} \geq 2 M \geq v,
$$


if $C \geq 3\left(1-e^{-1}\right)^{-1}$. On the bottom of $\aleph_{k}$ we have

$$
\Psi_{k} \geq C M\left(1-e^{-s)}\right)-\gamma s_{n} \geq 2 M \geq v,
$$

provided that

$$
C \geq \frac{2 M+\gamma s}{M\left(1-e^{-s}\right)}
$$

By direct calculation,

$$
\begin{aligned}
& \Psi_{k, x_{j}}=k C M \eta_{k} \frac{x_{j}-y_{j}}{|x-y|}, \\
& \Psi_{k, x_{l} x_{j}}=-k^{2} C M \eta_{k} \frac{\left(x_{j}-y_{j}\right)\left(x_{l}-y_{l}\right)}{|x-y|^{2}}+k C M \eta_{k} \frac{1}{|x-y|^{2}}\left[\delta_{j l}|x-y|-\frac{\left(x_{j}-y_{j}\right)\left(x_{l}-y_{l}\right)}{|x-y|}\right] \text {, } \\
& \Psi_{k, x_{i} x_{i}}=-k^{2} C M \eta_{k}+k C M \eta_{k} \frac{1}{|x-y|^{2}}(N|x-y|-|x-y|)=k C M \eta_{k}\left(-k+\frac{N-1}{|x-y|}\right) \text {, } \\
& {\left[\left(\left|\nabla \Psi_{k}\right|^{2}+\frac{1}{n}\right)^{\frac{p-2}{2}} \Psi_{k, x_{i}}\right]_{x_{i}}} \\
& =\frac{p-2}{2}\left(\left|\nabla \Psi_{k}\right|^{2}+\frac{1}{n}\right)^{\frac{p-4}{2}} 2 \Psi_{k, x_{l}} \Psi_{k, x_{l} x_{i}} \Psi_{k, x_{i}}+\left(\left|\nabla \Psi_{k}\right|^{2}+\frac{1}{n}\right)^{\frac{p-2}{2}} \Psi_{k, x_{i} x_{i}} \\
& =(p-2)\left[\left(k C M \eta_{k}\right)^{2}+\frac{1}{n}\right]^{\frac{p-4}{2}}\left(k C M \eta_{k}\right)^{2} \frac{\left(x_{j}-y_{j}\right)\left(x_{l}-y_{l}\right)}{|x-y|^{2}} \\
& \cdot\left\{-k^{2} C M \eta_{k} \frac{\left(x_{j}-y_{j}\right)\left(x_{l}-y_{l}\right)}{|x-y|^{2}}+\frac{k C M \eta_{k}}{|x-y|^{2}}\left[\delta_{j l}|x-y|-\frac{\left(x_{j}-y_{j}\right)\left(x_{l}-y_{l}\right)}{|x-y|}\right]\right\} \\
& +\left[\left(k C M \eta_{k}\right)^{2}+\frac{1}{n}\right]^{\frac{p-2}{2}} k C M \eta_{k}\left(-k+\frac{N-1}{|x-y|}\right) \\
& =G_{n} k C M \eta_{k}\left[-k+\frac{1}{|x-y|^{2}}(|x-y|-|x-y|)\right] \\
& +\left[\left(k C M \eta_{k}\right)^{2}+\frac{1}{n}\right]^{\frac{p-2}{2}} k C M \eta_{k}\left(-k+\frac{N-1}{|x-y|}\right) \\
& =-G_{n} k^{2} C M \eta_{k}+\left[\left(k C M \eta_{k}\right)^{2}+\frac{1}{n}\right]^{\frac{p-2}{2}} k C M \eta_{k}\left(-k+\frac{N-1}{|x-y|}\right) \text {, }
\end{aligned}
$$

where $G_{n}=(p-2)\left[\left(k C M \eta_{k}\right)^{2}+\frac{1}{n}\right]^{\frac{p-4}{2}}\left(k C M \eta_{k}\right)^{2}$.

$$
\begin{aligned}
\Psi_{k, t} & -\operatorname{div}\left[\left(d^{\alpha}+\frac{1}{n}\right)\left(\left|\nabla \Psi_{k}\right|^{2}+\frac{1}{n}\right)^{\frac{p-2}{2}} \nabla \Psi_{k}\right] \\
= & -C M e^{-k(|x-y|-1)} e^{t}+\gamma-\alpha d^{\alpha-1} d_{x_{i}}\left[\left(\left|\nabla \Psi_{k}\right|^{2}+\frac{1}{n}\right)^{\frac{p-2}{2}} \Psi_{k, x_{i}}\right] \\
& -\left(d^{\alpha}+\frac{1}{n}\right)\left[\left(\left|\nabla \Psi_{k}\right|^{2}+\frac{1}{n}\right)^{\frac{p-2}{2}} \Psi_{k, x_{i}}\right]_{x_{i}}
\end{aligned}
$$




$$
\begin{aligned}
= & -C M e^{-k(|x-y|-1)} e^{t}+\gamma-\alpha d^{\alpha-1} d_{x_{i}}\left[\left(\left|k C M \eta_{k}\right|^{2}+\frac{1}{n}\right)^{\frac{p-2}{2}} k C M \eta_{k} \frac{x_{i}-y_{i}}{|x-y|}\right] \\
& -\left(d^{\alpha}+\frac{1}{n}\right)\left\{-G_{n} k^{2} C M \eta_{k}+\left[\left(k C M \eta_{k}\right)^{2}+\frac{1}{n}\right]^{\frac{p-2}{2}} k C M \eta_{K}\left(-k+\frac{N-1}{|x-y|}\right)\right\} \\
\geq & -C M e^{-k(|x-y|-1)} e^{t}+\gamma-\alpha d^{\alpha-1}\left(\left|k C M \eta_{k}\right|^{2}+\frac{1}{n}\right)^{\frac{p}{2}} \\
& +\left(d^{\alpha}+\frac{1}{n}\right) G_{n} k^{2} C M \eta_{k}+k\left(d^{\alpha}+\frac{1}{n}\right)\left(\left|k C M \eta_{k}\right|^{2}+\frac{1}{n}\right)^{\frac{p}{2}} \\
& -(N-1)\left(d^{\alpha}+\frac{1}{n}\right)\left(\left|k C M \eta_{k}\right|^{2}+\frac{1}{n}\right)^{\frac{p-2}{2}},
\end{aligned}
$$

where we have used the facts that $\left|d_{x_{i}}\right| \leq 1, \alpha \geq 1$.

Clearly, if we choose $k$ large enough, then we have

$$
\Psi_{k, t}-\operatorname{div}\left[\left(d^{\alpha}+\frac{1}{n}\right)\left(\left|\nabla \Psi_{k}\right|^{2}+\frac{1}{n}\right)^{\frac{p-2}{2}} \nabla \Psi_{k}\right] \geq 0 .
$$

It follows by the comparison principle that the solution of the problem (3.6)-(3.8) $v$, $v \leq \Psi_{k}$ in $\aleph_{k}$. In particular, $\forall 0<x_{N}<\frac{1}{k}$, due to the interior regularity of the solution of (1.1), which we have discussed in Section 2, we have

$$
\begin{aligned}
u\left(0,0, \ldots, 0_{N-1}, x_{N}, 0\right) & =\lim _{n \rightarrow \infty} v\left(0,0, \ldots, 0_{N-1}, x_{N}, 0\right) \\
& \leq \Psi_{k}\left(0,0, \ldots, 0_{N-1}, x_{N}, 0\right) \\
& =C M\left(1-e^{-k x_{N}}\right) \leq k C M x_{N} .
\end{aligned}
$$

Therefore there exists a constant $k$ depending only upon $N$, such that

$$
u(x, t) \leq k M \operatorname{dist}(x, \partial \Omega)
$$

for all $x \in \Omega$ such that $d(x)=\operatorname{dist}(x, \partial \Omega) \leq \frac{1}{k}$. On the other hand, if $\operatorname{dist}(x, \partial \Omega)>\frac{1}{k}$, we have

$$
u(x, t) \leq M \leq k C M \operatorname{dist}(x, \partial \Omega)
$$

Thus (3.5) holds in both cases.

Estimates below: Let $u$ be a nonnegative bounded solution of (1.1) with homogeneous boundary value condition in the sense of Definition 3.1,

$$
u \leq M
$$

for some $M>0$. For $r>0$ let

$$
\begin{aligned}
& \Omega_{r} \equiv\{x \in \Omega \mid d(x, \partial \Omega) \geq r\}, \\
& \Omega_{r, t} \equiv \Omega_{r} \times[s, t], \quad \forall s<t \leq T,
\end{aligned}
$$


and

$$
\mu(r) \equiv \inf _{(x, \tau) \in \Omega r, t} u(x, \tau)
$$

For $0<s<t<T$, let

$$
r(M, s, t)=\rho_{0} \min \{1, \sqrt{t-s}\}
$$

Here the constant $\rho_{0}$ makes the inclusion $\Omega \cap\left\{|x|<\rho_{0}\right\} \subset\left\{x_{N}>0\right\}$ true as before.

Now, we estimate $u$ below, near the boundary $\partial \Omega$.

Theorem 3.3 If the hypothesis of Theorem 3.2 is true, then for $\forall 0<s<t<T, \forall x \in \Omega$, $d(x, \partial \Omega) \leq r(M, s, t)$, the inequality

$$
u(x, t) \geq \mu(r(M, s, t)) \operatorname{dist}(x, \partial \Omega) .
$$

holds.

The main idea is to construct a subsolution of the equation.

Proof Fix $\left(x_{0}, t_{0}\right) \in \partial \Omega \times(s, T)$ and let $\mu_{0} \equiv \mu\left(r\left(M, s, t_{0}\right)\right)$. After flattening of $\partial \Omega$ near $x_{0}$, we may assume that $\left(x_{0}, t_{0}\right) \equiv(0,0)$ as before. Introduce the point

$$
\bar{y} \equiv\left(0,0, \ldots, 0_{N-1}, 1+\frac{1}{k}\right)
$$

and the domain

$$
\bar{\aleph}_{k n}=\left\{(x, t): 0<x_{N}<\frac{1}{k}, 1<|x-\bar{y}|<1+\frac{1}{k},-s_{n} \leq t \leq 0\right\} \subset B_{\rho_{0}}^{+} \times[-s, 0],
$$

where

$$
\frac{1}{k}=r\left(M, s, t_{0}\right)=\rho_{0} \min \{1, \sqrt{s}\}
$$

Consider $-s<-s_{n}<t \leq 0$

$$
\begin{aligned}
& \frac{\partial v}{\partial t}=\operatorname{div}\left[\left(d^{\alpha}+\frac{1}{n}\right)\left(|\nabla v|^{2}+\frac{1}{n}\right)^{\frac{p-2}{2}} \nabla v\right], \quad(x, t) \text { in } \bar{\aleph}_{k n} . \\
& v_{n}\left(x,-s_{n}\right)=u_{n}\left(x,-s_{n}\right), \quad\left(x, s_{n}\right) \in \bar{\aleph}_{k n}, \\
& v(x, t)=\frac{1}{n}+u_{n}(x, t), \quad(x, t) \in \partial \bar{B}_{k} \times\left[-s_{n}, 0\right],
\end{aligned}
$$

where $u_{n}$ is the nonnegative solution of the problem (3.1)-(3.3), $s_{n} n$ is small enough, and $\bar{B}_{k}=\left\{x: 0<x_{N}<\frac{1}{k}, 1<|x-y|<1+\frac{1}{k}\right\}$. Also by the comparison theorem [8, p.119], we have

$$
v \geq u_{n}
$$




\section{Consider the function}

$$
\bar{\eta}_{k}(x, t)=e^{-k(|x-\bar{y}|-1)} e^{\frac{t}{s}}
$$

and construct the barrier

$$
\bar{\Psi}_{k}(x, t)=\mu_{0}\left(\bar{\eta}_{k}(x, t)-e^{-1}\right)_{+}-\gamma t,
$$

where $\gamma=\gamma\left(s, \mu_{0}, k\right)$ is large enough constant chosen later. Let us show that $v \geq \bar{\Psi}_{k}$ on the parabolic boundary of $\bar{\aleph}_{k}$. On the portion $\left\{|x-\bar{y}|=1+\frac{1}{k}\right\} \times\left[-s_{n}, 0\right]$ we have $\bar{\Psi}_{k}=$ $-\gamma t \leq \gamma s_{n} \leq \frac{1}{n} \leq v$. On the portion lying on the hyperplane $\left\{x_{N}=\frac{1}{k}\right\}$ one checks that $\bar{\Psi}_{k} \leq \mu_{0} \leq u \leq v$. On the bottom of $\bar{\aleph}_{k}$, we have

$$
\left.\bar{\Psi}_{k}\right|_{t=-s_{n}} \leq \mu_{0}\left(e^{-k(|x-\bar{y}|-1)}-1\right)_{+}+\gamma s_{n} \leq 0 .
$$

By direct calculation

$$
\begin{aligned}
& \bar{\Psi}_{k, x_{j}}=-k \mu_{0} \bar{\eta}_{k} \frac{x_{j}-y_{j}}{|x-y|}, \\
& \frac{\partial \bar{\Psi}}{\partial t}=\mu_{0} \bar{\eta}_{k} \frac{1}{s_{n}}-\gamma,
\end{aligned}
$$

and

$$
\begin{aligned}
& \bar{\Psi}_{k, x_{l} x_{j}}=k^{2} \mu_{0} \bar{\eta}_{k} \frac{\left(x_{j}-\bar{y}_{j}\right)\left(x_{l}-\bar{y}_{l}\right)}{|x-\bar{y}|^{2}}-k \mu_{0} \bar{\eta}_{k} \frac{1}{|x-\bar{y}|^{2}}\left[\delta_{j l}|x-\bar{y}|-\frac{\left(x_{j}-\bar{y}_{j}\right)\left(x_{l}-\bar{y}_{l}\right)}{|x-\bar{y}|}\right], \\
& \Psi_{k, x_{i} x_{i}}=k^{2} \mu_{0} \bar{\eta}_{k}-k \mu_{0} \bar{\eta}_{k} \frac{1}{|x-\bar{y}|^{2}}[N|x-\bar{y}|-|x-\bar{y}|]=k \mu_{0} \bar{\eta}_{k}\left(k-\frac{N-1}{|x-\bar{y}|}\right) \text {, } \\
& {\left[\left(\left|\nabla \bar{\Psi}_{k}\right|^{2}+\frac{1}{n}\right)^{\frac{p-2}{2}} \bar{\Psi}_{k, x_{i}}\right]_{x_{i}}} \\
& =\frac{p-2}{2}\left(\left|\nabla \bar{\Psi}_{k}\right|^{2}+\frac{1}{n}\right)^{\frac{p-4}{2}} 2 \bar{\Psi}_{k, x_{l}} \bar{\Psi}_{k, x_{l} x_{i}} \bar{\Psi}_{k, x_{i}}+\left(\left|\nabla \Psi_{k}\right|^{2}+\frac{1}{n}\right)^{\frac{p-2}{2}} \Psi_{k, x_{i} x_{i}} \\
& =(p-2)\left[\left(k \mu_{0} \bar{\eta}_{k}\right)^{2}+\frac{1}{n}\right]^{\frac{p-4}{2}}\left(k \mu_{0} \bar{\eta}_{k}\right)^{2} \frac{\left(x_{i}-\bar{y}_{i}\right)\left(x_{l}-\bar{y}_{l}\right)}{|x-\bar{y}|^{2}} \\
& \cdot\left\{k^{2} \mu_{0} \bar{\eta}_{k} \frac{\left(x_{i}-\bar{y}_{i}\right)\left(x_{l}-\bar{y}_{l}\right)}{|x-\bar{y}|^{2}}+\frac{k \mu_{0} \bar{\eta}_{k}}{|x-\bar{y}|^{2}}\left[\delta_{i l}|x-\bar{y}|-\frac{\left(x_{j}-\bar{y}_{j}\right)\left(x_{l}-\bar{y}_{l}\right)}{|x-\bar{y}|}\right]\right\} \\
& +\left[\left(k \mu_{0} \bar{\eta}_{k}\right)^{2}+\frac{1}{n}\right]^{\frac{p-2}{2}} k \mu_{0} \bar{\eta}_{k}\left(k-\frac{N-1}{|x-\bar{y}|}\right) \\
& =\bar{G}_{n} k \mu_{0} \bar{\eta}_{k}\left[k-\frac{1}{|x-\bar{y}|^{2}}(|x-\bar{y}|-|x-\bar{y}|)\right] \\
& +\left[\left(k \mu_{0} \bar{\eta}_{k}\right)^{2}+\frac{1}{n}\right]^{\frac{p-2}{2}} k \mu_{0} \bar{\eta}_{k}\left(k-\frac{N-1}{|x-\bar{y}|}\right) \\
& =G_{n} k^{2} \mu_{0} \bar{\eta}_{k}+\left[\left(k \mu_{0} \eta_{k}\right)^{2}+\frac{1}{n}\right]^{\frac{p-2}{2}} k \mu_{0} \bar{\eta}_{k}\left(k-\frac{N-1}{|x-y|}\right) \text {, }
\end{aligned}
$$


where $\bar{G}_{n}=(p-2)\left[\left(k \mu_{0} \bar{\eta}_{k}\right)^{2}+\frac{1}{n}\right]^{\frac{p-4}{2}}\left(k \mu_{0} \bar{\eta}_{k}\right)^{2}$. We have

$$
\begin{aligned}
\bar{\Psi}_{k, t} & -\operatorname{div}\left[\left(d^{\alpha}+\frac{1}{n}\right)\left(\left|\nabla \bar{\Psi}_{k}\right|^{2}+\frac{1}{n}\right)^{\frac{p-2}{2}} \nabla \bar{\Psi}_{k}\right] \\
= & \mu_{0} e^{-k(|x-y|-1)} e^{\frac{t}{s_{n}}} \frac{1}{s_{n}}-\gamma-\alpha d^{\alpha-1} d_{x_{i}}\left[\left(\left|\nabla \bar{\Psi}_{k}\right|^{2}+\frac{1}{n}\right)^{\frac{p-2}{2}} \bar{\Psi}_{k, x_{i}}\right] \\
& -\left(d^{\alpha}+\frac{1}{n}\right)\left[\left(\left|\nabla \bar{\Psi}_{k}\right|^{2}+\frac{1}{n}\right)^{\frac{p-2}{2}} \bar{\Psi}_{k, x_{i}}\right]_{x_{i}} \\
= & \mu_{0} e^{-k(|x-y|-1)} \frac{\frac{t}{s_{n}}}{\frac{1}{s_{n}}}-\gamma-\alpha d^{\alpha-1} d_{x_{i}}\left[\left(\left|k \mu_{0} \bar{\eta}_{k}\right|^{2}+\frac{1}{n}\right)^{\frac{p-2}{2}} k \mu_{0} \bar{\eta}_{k} \frac{x_{i}-\bar{y}_{i}}{|x-\bar{y}|}\right] \\
& -\left(d^{\alpha}+\frac{1}{n}\right)\left\{\bar{G}_{n} k^{2} \mu_{0} \bar{\eta}_{k}+\left[\left(k \mu_{0} \bar{\eta}_{k}\right)^{2}+\frac{1}{n}\right]^{\frac{p-2}{2}} k \mu_{0} \bar{\eta}_{k}\left(k-\frac{N-1}{|x-\bar{y}|}\right)\right\} \\
\leq & \mu_{0} e^{-k(|x-y|-1)} e^{\frac{t}{s_{n}}} \frac{1}{s_{n}}-\gamma+\alpha d^{\alpha-1}\left(\left|k \mu_{0} \eta_{k}\right|^{2}+\frac{1}{n}\right)^{\frac{p}{2}} \\
& -\left(d^{\alpha}+\frac{1}{n}\right) \bar{G}_{n} k^{2} \mu_{0} \bar{\eta}_{k}-k\left(d^{\alpha}+\frac{1}{n}\right)\left|k \mu_{0} \bar{\eta}_{k}\right|^{p-1} \\
& +(N-1)\left(d^{\alpha}+\frac{1}{n}\right)\left(\left|k \mu_{0} \eta_{k}\right|^{2}+\frac{1}{n}\right)^{\frac{p-2}{2}},
\end{aligned}
$$

where we have used the fact that $\left|d_{x_{i}}\right| \leq 1$.

Clearly, if we choose $\gamma$ large enough, then we have

$$
\Psi_{k, t}-\operatorname{div}\left[\left(d^{\alpha}+\frac{1}{n}\right)\left(\left|\nabla \Psi_{k}\right|^{2}+\frac{1}{n}\right)^{\frac{p-2}{2}} \nabla \Psi_{k}\right] \leq 0 .
$$

It follows from the comparison principle that $v \geq \bar{\Psi}_{k}$ in $\bar{\aleph}_{k}$. In particular, $\forall 0<x_{N}<\frac{1}{k}$,

$$
v\left(0,0, \ldots, 0_{N-1}, x_{N}, 0\right) \geq \frac{\mu_{0}}{e}\left(e^{k x_{N}}-1\right) \geq \frac{\mu_{0} k}{e} x_{N},
$$

also, due to the interior regularity of the solution (1.1), which we have discussed in Section $2, \forall x \in \Omega$ such that $\operatorname{dist}(x, \partial \Omega) \leq r(M, s, t)$, let $n \rightarrow \infty$. We have

$$
\begin{aligned}
u(x, t) & \geq \frac{k}{e} \mu(r(M, s, t)) \operatorname{dist}(x, \partial \Omega) \\
& \geq \mu(r(M, s, t)) \operatorname{dist}(x, \partial \Omega) .
\end{aligned}
$$

Theorem 3.3 is proved.

Remark 3.4 The method of estimates near the boundary we used here is classical, which strongly depends on the construction of special barrier functions. The shortcoming of such a technique is evident even in the framework of an evolutionary $p$-Laplacian equation (1.2) itself (see [9] etc.). For the diffusion (1.1) with $\alpha<1$, whether the estimate (3.5) or (3.10) is true or not is a problem to be probed in the future. To solve this open question, it strongly depends on how to extend the Harnack estimates to the case of parabolic 
equations with the full quasilinear structure, as it happens when $p=2$. Results of this kind would probably require a new method independent of local representations and local subsolutions. Whenever developed, such a technique may parallel the discovery of the Moser estimates [10], based on real and harmonic analysis tools, versus the estimates by Hadamards [11] and Pini [12], based on local representations.

\section{Estimates of the gradient near the boundary}

In the last section, we are concerned with the estimates of the gradient of the solution near the boundary. We will prove the following theorem.

Theorem 4.1 Supposed that $p>\max \left\{1, \frac{2 N}{N+1}\right\}, \alpha>0$. Supposed that the points $x \in \Omega$ is close enough to the boundary $\partial \Omega$, then for the unique weak solution $u$ of (1.1) with the homogeneous boundary value in the sense of Definition 3.1, there exists a constant $\gamma$, such that for all $(x, t) \in \Omega_{T}, d(x)=\operatorname{dist}(x, \partial \Omega)$ is small enough, we have

$$
|\nabla u(x, t)| \leq \gamma d^{-\alpha}
$$

Remark 4.2 The estimate (4.1) we get here is not so beautiful as the result in [13]. If one refers to Theorem 1.3 of [13], in which $\alpha=0, p>3$, one has

$$
|\nabla u(x, t)| \leq \gamma d(x)
$$

Proof of Theorem 4.1 For $\left(x_{0}, t_{0}\right) \in \Omega_{T}$, consider the cylindrical domain

$$
Q_{0}=\left\{\left|x-x_{0}\right|<\frac{1}{2} d_{0}\right\} \times\left\{t_{0}-\frac{d_{0}^{p}}{2^{p} u\left(x_{0}, t_{0}\right)^{2-p}}, t_{0}+\frac{d_{0}^{p}}{2^{p} u\left(x_{0}, t_{0}\right)^{2-p}}\right\} .
$$

Since we are concerned with those points near the boundary, without loss of generality, we may assume $d_{0}$ is small enough such that

$$
t_{0}-\frac{d_{0}^{p}}{2^{p} u\left(x_{0}, t_{0}\right)^{2-p}}>0
$$

and that $Q_{0} \subset \Omega \times(0, T)$. Introduce the change of variables

$$
\xi=\frac{2\left(x-x_{0}\right)}{d_{0}}, \quad \tau=\frac{2^{p}\left(t-t_{0}\right) u^{p-2}\left(x_{0}, t_{0}\right)}{d_{0}^{p}}
$$

and the new function

$$
\psi(\xi, \tau)=\frac{u(x, t)}{u\left(x_{0}, t_{0}\right)}
$$

This maps $Q_{0}$ into the box $Q_{1}=\{|\psi|<1\} \times\{|\tau|<1\}$. Moreover, $\psi$ satisfies

$$
\psi_{\tau}-\operatorname{div}\left(d_{1}^{\alpha}|\nabla \psi|^{p-2} \nabla \psi\right)=0
$$


in which $d_{1}(\xi, \tau)=d(x, t)$, but it is not the distance function of $(\xi, \tau)$ to the boundary of $Q_{1}$. If $\alpha<p-1$, in such a box, according to Theorems 2.3-3.3, we have

$$
\gamma_{1} \frac{d(x, t)}{u\left(x_{0}, t_{0}\right)} \leq \psi(\xi, \tau)=\frac{u(x, t)}{u\left(x_{0}, t_{0}\right)} \leq \gamma_{2} \frac{d(x, t)}{u\left(x_{0}, t_{0}\right)} .
$$

However, we do not use this estimate in the following proof.

Now, let

$$
w=v^{\frac{p+2 \beta}{4}}=|\nabla \psi|^{\frac{p+2 \beta}{2}} .
$$

Similar to the discussion of $(2.10)$ in Theorem 2.3, for simplicity, we still denote $(\xi, \tau)$ as $(x, t)$ in the following, and we get

$$
\begin{aligned}
& \sup _{T_{n}<t<T} \int_{B_{n}}\left(\xi_{n}^{\frac{2}{\lambda}} w\right)^{\lambda}(x, t) d x+\iint_{Q_{n}}\left(\xi_{n}^{\frac{2}{\lambda}}|\nabla w|\right)^{2} d x d t \\
& \quad \leq \frac{C}{d_{0}^{\alpha}}\left[\left(1+\frac{2^{2 n}}{\rho^{2}}\right) \iint_{Q_{n}} w^{2} d x d t+\frac{2^{n}}{t_{0}} \iint_{Q_{n}} w^{\lambda} d x d t\right],
\end{aligned}
$$

where $t_{0}=\tau_{0}+2, \tau_{0} \in(-1,1), Q_{n}=B_{n} \times\left(T_{n}, T\right) \subset\{|\psi|<1\} \times\{|\tau|<1\}$. By Lemma 2.1 and (4.9),

$$
\iint_{Q_{n+1}} w^{2+\frac{2 \lambda}{N}} d x d \tau \leq \frac{C}{d_{0}^{\alpha}}\left[\left(1+\frac{2^{2 n}}{\rho^{2}}\right) \iint_{Q_{n}} w^{2} d x d t+\frac{2^{n}}{t_{0}} \iint_{Q_{n}} w^{\lambda} d x d t\right]
$$

which implies that

$$
\begin{aligned}
& \iint_{Q_{n+1}} v^{\frac{p+2 \beta}{2}+\frac{2 \beta+2}{N}} d x d \tau \\
& \quad \leq \frac{C}{d_{0}^{\alpha}}\left[\left(1+\frac{2^{2 n}}{\rho^{2}}\right) \iint_{Q_{n}} v^{\frac{p+2 \beta}{2}} d x d \tau+\frac{2^{n}}{t_{0}} \iint_{Q_{n}} v^{\beta+1}\right]^{1+\frac{2}{N}},
\end{aligned}
$$

where $k=1+\frac{2}{N}$. By choosing $2 \beta=k^{n}-2$, the above formula can be changed into

$$
\begin{aligned}
& \iint_{Q_{n+1}} v^{\frac{p-2}{2}+\frac{k^{n+1}}{2}} d x d \tau \\
& \quad \leq \frac{C}{d_{0}^{\alpha}}\left[\left(1+\frac{2^{2 n}}{\rho^{2}}\right) \iint_{Q_{n}} v^{\frac{p-2}{2}+\frac{k^{n}}{2}} d x d \tau+\frac{2^{n}}{t_{0}} \iint_{Q_{n}} v^{\frac{k^{n}}{2}} d x d \tau\right]^{k} .
\end{aligned}
$$

If

$$
\frac{2^{n}}{t_{0}} \iint_{Q_{n}} v^{\frac{k^{n}}{2}} d x d \tau \geq\left(1+\frac{2^{2 n}}{\rho^{2}}\right) \iint_{Q_{n}} v^{\frac{p-2}{2}+\frac{k^{n}}{2}} d x d \tau,
$$

then by the Hölder inequality, we see that

$$
\iint_{Q_{n}} v^{\frac{p-2}{2}+\frac{k^{n}}{2}} d x d \tau \leq \frac{C}{d_{0}^{\alpha}}\left(\frac{\rho^{2}}{t_{0}}\right)^{\frac{\left(p-2+k^{n}\right)}{(p-2)}} \operatorname{mes} Q_{n},
$$


which implies that

$$
\sup _{B_{\rho} \times\left(\frac{\tau_{0}}{2}, T\right)} v \leq \frac{C}{d_{0}^{\alpha}}\left(\frac{\rho^{2}}{t_{0}}\right)^{\frac{2}{(p-2)}} .
$$

If (4.12) is not true, we have

$$
\iint_{Q_{n+1}} v^{\frac{p-2}{2}+\frac{k^{n+1}}{2}} d x d \tau \leq \frac{C}{d_{0}^{\alpha}}\left[\left(1+\frac{2^{2 n}}{\rho^{2}}\right) \iint_{Q_{n}} v^{\frac{p-2}{2}+\frac{k^{n}}{2}} d x d \tau\right]^{k} .
$$

By Lemma 2.2,

$$
\sup _{Q_{n}} v \leq \frac{C}{d_{0}^{\alpha}} \rho^{-(N+2)} \iint_{Q_{n_{0}}} v^{\frac{p-2}{2}+\frac{k^{n_{0}}}{2}} d x d \tau,
$$

where $n_{0}$ is a positive integer which makes $k^{n_{0}}>2$ hold. Then, according to (2.5), which is still true, and because the constant $C$ is independent of $d_{0}$, we have

$$
\sup _{Q_{n}} v \leq \frac{C}{d_{0}^{\alpha}} \rho^{-(N+2)}
$$

Especially, we choose $\left(\psi_{0}, \tau_{0}\right)=(0,0), \rho=\frac{1}{2}$, from (4.13)-(4.14), we have

$$
|\nabla \psi(0,0)| \leq \frac{C}{d_{0}^{\alpha}} .
$$

Now in terms of $u$, it means that

$$
\left|\nabla u\left(x_{0}, t_{0}\right)\right| \leq \gamma d_{0}^{-\alpha} .
$$

The theorem is proved.

\section{Competing interests}

The authors declare that they have no competing interests.

Authors' contributions

All authors contributed equally to the writing of this paper. All authors read and approved the final manuscript.

\section{Author details}

${ }^{1}$ School of Applied Mathematics, Xiamen University of Technology, Xiamen, 361024, P.R. China. ${ }^{2}$ Department of Mathematics, Yan-En University, Quanzhou, 362014, P.R. China.

\section{Acknowledgements}

The paper is supported by NSF of China (no. 11371297), supported by SF of Xiamen University of Technology, China.

Received: 28 October 2013 Accepted: 14 July 2014 Published: 18 Aug 2014

\section{References}

1. Wu, Z, Zhao, J, Yun, J, Li, F: Nonlinear Diffusion Equations, World Scientific, Singapore (2001)

2. Di Benedetto, E: Degenerate Parabolic Equations. Spring, New York (1993)

3. Vazquez, JL: Smoothing and Decay Estimates for Nonlinear Diffusion Equations. Oxford University Press, London (2006)

4. Yin, J, Wang, C: Properties of the boundary flux of a singular diffusion process. Chin. Ann. Math., Ser. B 25, 175-182 (2004) 
5. Di Benedetto, E, Kwong, Y, Verspi, V: Local space analyticity and asymptotic behavior of solutions of certain singular parabolic equations. Indiana Univ. Math. J. 40, 741-765 (1991)

6. Yin, J, Wang, C: Evolutionary weighted p-Laplacian with boundary degeneracy. J. Differ. Equ. 237, 421-445 (2007)

7. Karcher, H: Riemannian comparison construction. In: Global Differential Geometry. MAA Stud. Math., vol. 27, pp. 170-222 (1989)

8. Liankun, G: Partial Differential Equations of Second-Order Parabolic. Xiamen University Publishing, Xiamen (2002) (in Chinese)

9. Di Benedetto, E, Urbano, JM, Vespri, V: Current issues on singular and degenerate evolution equations. In: Evolutionary Equations. Handbook of Differential Equations, vol. 1, pp. 169-286. North-Holland, Amsterdam (2004)

10. Moser, J: A Harnack inequality foe parabolic differential equations. Commun. Pure Appl. Math. 17, 101-134 (1964)

11. Hadamard, J: Extension á l'équation de la chaleur d'un théoréme de A. Harnack. Rend. Circ. Mat. Palermo 23, 337-346 (1954)

12. Pini, B: Sulla soluzione generalizzata di Winener per il primo problema di valori al contorno nel parabolico. Rend. Semin. Mat. Univ. Padova 23, 422-434 (1954)

13. Manfredi, J, Vespri, V: Large time behavior of solutions to a class of doubly nonlinear parabolic equations. Electron. J. Differ. Equ. 1994, 02 (1994)

10.1186/1029-242X-2014-284

Cite this article as: Zhan and Xie: The boundary degeneracy of a singular diffusion equation. Journal of Inequalities and Applications 2014, 2014:284

\section{Submit your manuscript to a SpringerOpen ${ }^{\ominus}$ journal and benefit from:}

- Convenient online submission

- Rigorous peer review

- Immediate publication on acceptance

- Open access: articles freely available online

- High visibility within the field

- Retaining the copyright to your article 